\title{
A Secure and Efficient I dentity-Based Proxy Signcryption in Cloud Data Sharing
}

\author{
Negalign Wake Hundera ${ }^{1}$, Qian Mei ${ }^{1}$, Hu Xiong ${ }^{1}$ and Dagmawit Mesfin Geressu ${ }^{2}$ \\ ${ }^{1}$ School of Information and Software Engineering, University of Electronic Science and Technology of China \\ Chengdu China 610054. \\ [e-mail: nigaccna21@gmail.com] \\ ${ }^{2}$ School of Information Communication Engineering, University of Electronic Science and Technology of China \\ Chengdu China 610054. \\ *Corresponding author: Hu Xiong
}

Received April 21, 2019; revised July 6, 2019; accepted September 30, 2019; published January 31, 2020

\begin{abstract}
As a user in modern societies with the rapid growth of Internet environment and more complicated business flow processes in order to be effective at work and accomplish things on time when the manager of the company went for a business trip, he/she need to delegate his/her signing authorities to someone such that, the delegatee can act as a manager and sign a message on his/her behalf. In order to make the delegation process more secure and authentic, we proposed a secure and efficient identity-based proxy signcryption in cloud data sharing (SE-IDPSC-CS), which provides a secure privilege delegation mechanism for a person to delegate his/her signcryption privilege to his/her proxy agent. Our scheme allows the manager of the company to delegate his/her signcryption privilege to his/her proxy agent and the proxy agent can act as a manager and generate signcrypted messages on his/her behalf using special information called "proxy key". Then, the proxy agent uploads the signcrypted ciphertext to a cloud service provider (CSP) which can only be downloaded, decrypted and verified by an authorized user at any time from any place through the Internet. Finally, the security analysis and experiment result determine that the proposed scheme outperforms previous works in terms of functionalities and computational time.
\end{abstract}

Keywords: proxy signcryption, proxy credential, proxy key, proxy signature, delegator, delegate. 


\section{Introduction}

The objective of public key cryptography (PKC) and digital signature is used to increase the privacy of the data by achieving the four important security requirements such as confidentiality, integrity, authentication, and non-repudiation. A traditional way of obtaining these four security goals is first to sign then apply the encryption algorithm on the message. Recently those most popular research areas such as cloud email systems, computer communications, a delegation of organizational power and electronic transactions need both security requirements. Because of the high computational cost and communication overhead, it's difficult to achieve all these goals simultaneously with the traditional approach. In 1997, Zheng [1] made the signcryption idea that accomplishes both confidentiality and authentication in a single reasonable step with better performance than the traditional methods. Later, many signcryption schemes have been proposed [2-9]. Some of these are proxy signcryption [6-8] which efficiently combines the idea of proxy signature with the signcryption scheme, and allows an entity to delegate its signcryption authority to a trusted agent on PKI framework. To solve the key management processes in PKI, the idea of ID-based cryptography (IBC) was developed by Shamir [10] in 1984. In ID-based cryptography, the recognized string (ASCII string ) or identity such as email addresses, postal code, social security number represents an individual or organization public key, while the private key of user's is generated by PKG from their identity information. Malone-Lee [11] extended the signcryption idea to an ID-based signcryption scheme. Ever since many ID-based signatures [12-15] and signcryption [16-20] schemes have been proposed. Their key objective is to decrease the computational costs and develop efficient ID-based signcryption schemes. Now let's consider the situation when the manager of a company went for a business trip for a short or long period of time, in order to be effective at work and accomplish things on time he/she must delegate his/her signcryption authority to a proxy signcryptor who can legitimately signcrypt on behalf of him/her. So, this kind of situation must fulfill all the security requirements and the delegation process must be done in a secure and authentic way. The basic idea of our SE-IDPSC-CS scheme is as follow; the manager of the company officially delegate his/her signcryption authorization to his/her proxy agent and the proxy agent act as a manager and generate signcrypted messages on his/her behalf by using special information called " proxy key". Then the proxy agent uploads the signcrypted ciphertext to a trusted cloud service provider (CSP). Finally, the authorized user can download, recover and verify its source and validity at any time from any place through the Internet. Recently, Li and Chen [20] made ID-based proxy signcryption model, but their scheme is not secure and proxy protected, because the delegator is the only one who generates the proxy key without the knowledge of the proxy agent and they simply added the proxy key on [20] signcryption algorithm, if the original signcrypter remove the proxy key he/she will recover the message. Chen et al. [22] presented a probably secure ID-based proxy signcryption model under CDHP and BDHP assumptions. Ming et al. [23] constructed an ID-based proxy signcryption model without random oracles. Zhou [24] developed secure ID-based generalized proxy signcryption without random oracles from bilinear pairings and $\mathrm{H}$ Yu [25] proposed an ID-based proxy signcryption protocol with UC. But still, now all the above schemes consume high computational cost. This paper explains a new secure and efficient identity-based proxy signcryption in cloud data sharing (SE-IDPSC-CS) which is more secure and efficient than the existing schemes. The design philosophy behind our proposed scheme is as follow, the 
manager of the company that is the original signcryptor officially delegate his/ her signcryption authority to proxy signcryptor, who then act as a manager and generate a signcrypted messages on his/her behalf and upload the signcrypted ciphertext to cloud service provider (CSP), it is a trusted server which supplies storage services and sends the signcrypted ciphertexts to an authorized user. Finally, an authorized user download, decrypt and confirm the source and validness of the message. We also proof the security of the scheme in terms of IND-IDPSC-CS-CCA2 and EF-IDPSC-CS-CMA under DBDH and CDH problems respectively. We organized the paper as follows. We define the preliminary work and the basic notations in section 3 . The details of the system model, the overall framework and the security definition are presented in section 4 . Section 5 , provide the details of the construction while section 6 and section 7 describe security proof and performance analysis respectively. Finally, we conclude the paper in sections 8.

\section{Related Work}

A proxy signcryption [6-8] is a cryptographic algorithm that merges the idea of signcryption and algorithm with a proxy signature. In cryptography, signcryption is a cryptographic algorithm that achieves the functionality of both confidentiality and authentication in a single reasonable step with better performance than a traditional approach. The first signcryption scheme was proposed by Y. Zheng [1] later, several signcryption schemes have been proposed [2-8]. Proxy signature model, allows an entity officially delegate his/her signing right to someone so that he/she can sign a message on his/her behalf. The first proxy signature was proposed by Mambo et al. [26]. Later many proxy signature schemes [27-28] have been proposed. To solve key controlling processes in PKI, the concept of ID-based cryptography (IBC) was developed by Shamir [10] in 1984. In ID-based cryptography, the well-known string (ASCII string ) or identity such as email address, postal code, social security number represents an individual or organization public key, while the secret key of the user's generated by PKG from their identity data. Later, several well-organized ID-based signatures [29-31] and ID-based schemes using pairings [32-33] have been proposed. Later many new ID-based signatures [34-35] and signcryption [18-20] schemes have been proposed. Malone-Lee [11] elaborated the signcryption idea to an ID-based signcryption scheme. Ever since many ID-based signcryption schemes have been proposed [15-20]. Recently, Li and Chen proposed an ID-based proxy signcryption scheme [21]. However, their scheme is not proxy protected and does not meet the unforgeability and forward security. In 2005, Wang and Cao [36] proposed an ID-based proxy signature and proxy signcryption scheme, which is based on [18] and is efficient than [21] in terms of computational point of view. Chen et al. [22] presented a probably secure ID-based proxy signcryption model under CDHP and BDHP assumptions. Ming et al. [23] constructed an ID-based proxy signcryption model without random oracles. Zhou [24] developed secure ID-based generalized proxy signcryption without random oracles from bilinear pairings and $\mathrm{H} \mathrm{Yu} \mathrm{[25]} \mathrm{proposed} \mathrm{an} \mathrm{ID-based} \mathrm{proxy} \mathrm{signcryption} \mathrm{protocol} \mathrm{with}$ UC. Later Many schemes have been proposed for efficient and secure data accessing [37-40]. In this paper, by combining the idea of ID-based signcryption and proxy signature schemes, we proposed a new secure and efficient identity-based proxy signcryption in cloud data sharing (SE-IDPSC-CS) scheme, which is secure and efficient than the above schemes. In this scheme, after validating the identity of the delegator the proxy agent creates valid signcrypted ciphertext and uploads it to a cloud service provider (CSP). Moreover, only the authorized user can download, decrypt and confirm the source and authenticity of the message. Compared to the above schemes our scheme archived the necessary security requirements. 


\section{Preliminaries}

In this section, we briefly define the bilinear pairings and notations.

\subsection{Bilinear Pairings}

Let $\mathbb{G}_{1}$ additive and $\mathbb{G}_{2}$ multiplicative cyclic groups having similar prime order $q$. Let $a, b \in Z_{q^{*}}$ and $\mathbb{G}_{1}$ is generated by $P$. A bilinear map $\hat{e}: \mathbb{G}_{1} \times \mathbb{G}_{1} \rightarrow \mathbb{G}_{2}$ has the following properties:

a. Bilinear: $(\mathrm{aP}, \mathrm{bQ})=(\mathrm{P}, \mathrm{Q})^{a b}$ for all $P, Q \in \mathbb{G}_{1}, a, b \in Z_{q^{*}}$.

b. Non-degenerate: $P, Q \in \mathbb{G}_{1}$ so that, $\hat{e}(P, Q) \neq 1$, where 1 is the identity of $\mathbb{G}_{2}$.

c. Computability: for all $P, Q \in \mathbb{G}_{1}, \hat{e}(P, Q)$ efficiently computable.

The revised Weil pairing and Tate pairing is acceptable applications [31]. The security of our EF-IDPSC-CS model depends on the following hard Diffie-Hellman problems. Given $\mathbb{G}_{1}$ additive and $\mathbb{G}_{2}$ multiplicative cyclic groups of the same prime order $q$. Let $a, b \in Z_{q^{*}}$ and $\mathbb{G}_{1}$ is generated by $P$, a bilinear map $\hat{e}: \mathbb{G}_{1} \times \mathbb{G}_{1} \rightarrow \mathbb{G}_{2}$ :

a. Computational Diffie-Hellman Problem (CDHP): The CDHP in $\mathbb{G}_{1}$ is to calculate $a b P$ given $(P, a P, b P)$ for $a, b \in Z_{q^{*}}$.

a. Decisional Bilinear Diffie-Hellman Problem (DBDHP): Assumed a set $(P, a P, b P, c P)$ and an element $h \in \mathbb{G}_{2}$, the DBDHP to decide whether $h=\hat{e}(P, P)^{a b c}$ or not. We define the benefit of the adversary $\mathcal{C}$ against the DBDHP like this: $\quad \operatorname{Adv}(\mathcal{C})=$

$\left|P_{a, b, c \in Z_{q^{*}}, h \in \mathbb{G}_{2}}[1 \leftarrow \mathcal{C}(a P, b P, c P, h)]-P_{a, b, c \in Z_{q^{*}}}\left[1 \leftarrow \mathcal{C}\left(a P, b P, c P, \hat{e}(P, P)^{a b c}\right)\right]\right|$

The DBDHP normally not harder than CDHP.

\subsection{Notations}

The notations used in this paper are listed in Table 1.

Table 1. Acronym and Description

\begin{tabular}{|c|c|c|}
\hline No & Acronym & Description \\
\hline \hline 1 & $S k_{a p}$ & Proxy Key \\
\hline 2 & USC & Unsigncryption \\
\hline 3 & $S_{p c}$ & Proxy Credential \\
\hline 4 & PSC & Proxy Signcryption \\
\hline 5 & PKeyGen & Proxy Key Generation \\
\hline 6 & PKG & Private Key Generator \\
\hline 7 & $C_{T}$ & Signcryption Ciphertext \\
\hline 8 & ID-PS & Identity-Based Proxy Signature \\
\hline 9 & ID-PE & Identity-Based Proxy Encryption \\
\hline 10 & ID-PSC & Identity-Based Proxy Signcryption \\
\hline 11 & DBDH & Decision Bilinear Diffie-Hellman \\
\hline 12 & CDH & Computation Diffie-Hellman \\
\hline 13 & DLP & Discrete logarithm problem \\
\hline 14 & PKI & Public key infrastructure \\
\hline 15 & PKC & Public key cryptography \\
\hline 16 & IBC & ID-base cryptography \\
\hline
\end{tabular}




\section{System model, Framework and Security definition}

In this section, we will define the system model, framework and security definition of the scheme.

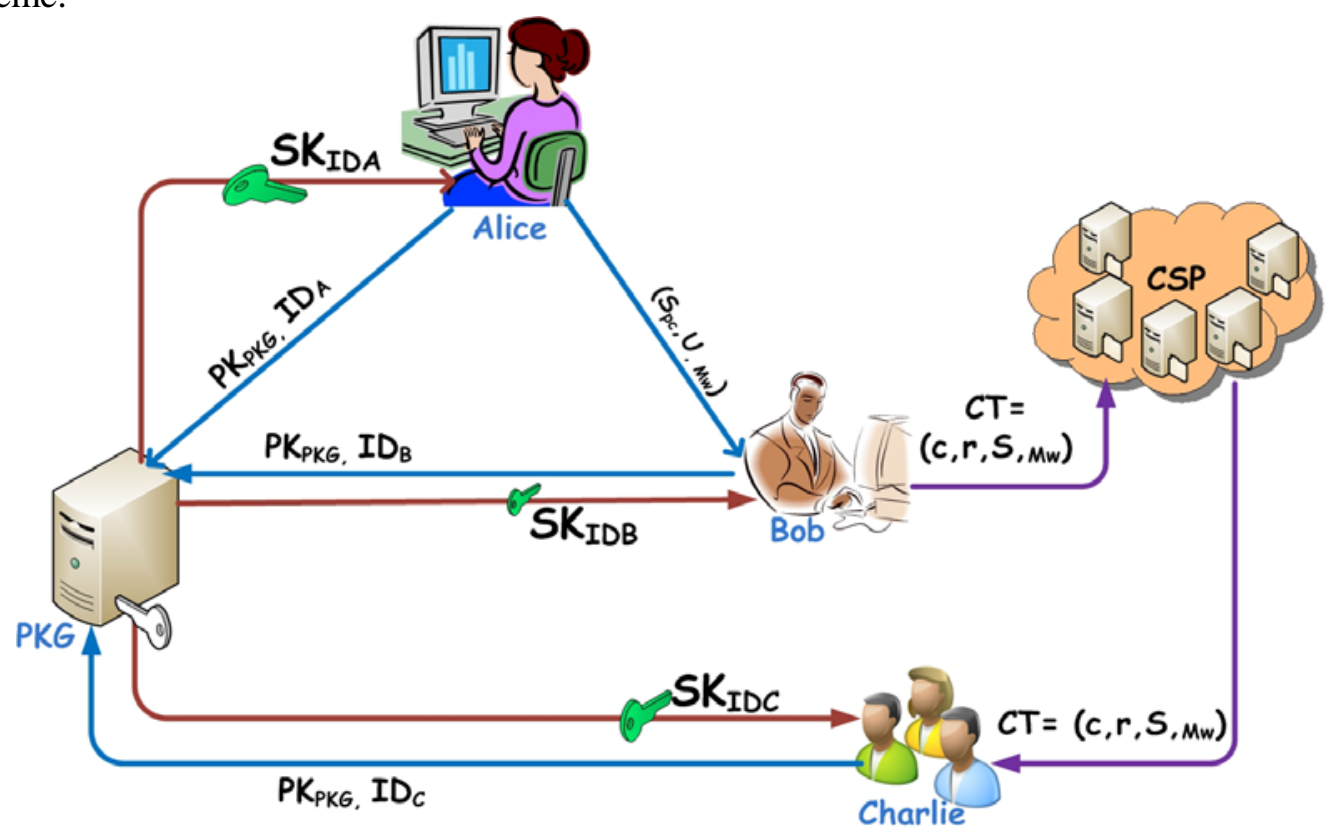

Fig. 1. Model of the SE-IDPSC-CS scheme.

\subsection{System Model}

According to Fig. 1, the architecture of SE-IDPSC-CS scheme consists of the following entities:

a. PKG: This is a trusted authority used to compute the user's private key from their identity information.

b. Delegator (Alice): This entity wants to delegate his/her signcryption authority to his/her proxy signcryptor (Bob).

c. Proxy Signcryptor (Bob): This is an entity that generates a signcrypted message on behalf of the delegator (Alice) by using the special information called "proxy key" and uploads it to a trusted cloud service provider (CSP).

d. Cloud service provider (CSP): This entity supplies the storage service and sends the signcrypted ciphertext to an authorized user.

e. Receiver (Charlie): An entity who download, wants to recover the message content and verify it's validity at any time, from anywhere through the Internet.

\subsection{Framework of SE-IDPSC-CS scheme}

Our scheme contains the following six algorithms, including the delegator (Alice) $Q_{I D_{A}}$, proxy signcryptor (Bob) $Q_{I D_{I}}$, receiver (Charlie) $Q_{I D_{C}}$ and the cloud service provider (CSP).

1. Setup: On input the security parameter $k, \mathbf{P K G}$ output the public parameters params, and keep the master key $s$ to itself.

2. Extract: On input, params, identity $I D$, and master secret key $s$ returns the private key $S_{I D}$ of $I D$, the PKG must transmit it to corresponding entities in a secure way. 
Assume $\left(Q_{I D_{A}}, S_{I D_{A}}\right)$ is delegator key pairs, $\left(Q_{I D_{B}}, S_{I D_{B}}\right)$ proxy signcryptor key pairs and $\left(Q_{I D_{C}}, S_{I D_{C}}\right)$ is receiver's key pairs.

3. Proxy Credential: This is an algorithm run by delegator that takes on input params, delegator private key $S_{I D_{A}}$, and warrant $m_{w}\left(m_{w}\right.$ contains the delegation time, identities of the delegator and proxy signcrypter), output a proxy credential $S_{p c}$ and sends $\left(S_{p c}, m_{w}\right)$ to a delegatee. There is a clear explanation of the delegation privileges and some common information about the delegator and proxy signcrypter in the warrant $m_{u}$ such that a receiver can use it as verification information.

4. PKeyGen: An algorithm run by proxy signcryptor (Bob) which takes params, warrant $m_{w}$, proxy credential $S_{p c}$ and delegatee private key $S_{I D_{13}}$ as input. Outputs a proxy key $S k_{a p}$.

5. Proxy Signcryption: An algorithm run by proxy signcryptor (Bob) that take on input params, the identity of the receiver $Q_{I D_{C}}$, the proxy key $S k_{a p}$, a warrant $m_{w}$, and a message $m$. Output a signcryption ciphertext $C_{T}$.

6. Unsigncryption: On input, public parameters params, ciphertext $C_{T}$ and $S_{I D_{C}}$ of the receiver, then confirm whether the ciphertext $C_{T}$ is correct, if "yes" continue and output the plaintext $m$, otherwise, output $\perp$.

\subsection{Security Definition}

We use a security game to describe the confidentiality and unforgeability of the message, here $\mathcal{C}$ is a challenger and $\mathcal{A}$ is an adversary respectively. We define two security models for these notions as follows:

Definition 1. We say that a SE-IDPSC-CS scheme is said to achieve the security requirement of IND-SE-IDPSC-CS-CCA2 if no polynomial-time adversaries who have a non-negligible advantage win in the following game:

Initial: $\mathcal{C}$ runs Setup algorithm to get params and $s$. Then send params to $\mathcal{A}$ and keeps $s$ to itself.

Phase 1: $\mathcal{A}$ adaptively performs several kinds of queries; each query may be dependent on the outcome of the previous queries:

Extract query QExtract(ID): $\mathcal{A}$ chooses an identity $I D$. $\mathcal{C}$ runs $\operatorname{Extract}(I D)$ and $S_{I D}$ to $\mathcal{A}$.

a. Proxy Credential query $\operatorname{QPC}\left(\right.$ params $\left., S_{I D_{i}}, m_{w}\right): \mathcal{A}$ issues a proxy credential request with respect to the delegatee. $\mathcal{C}$ returns a warrant $m_{w}$ and proxy credential $S_{p c}$.

b. PKeyGen query QPKeyGen $\left(S_{I D_{j}}, S_{p c}\right): \mathcal{A}$ selects two identities $I D_{i}, I D_{j}$, for a given identity $I D_{i}$ and $I D_{j}, \mathcal{C}$ first runs the $Q P C$ query to get $S_{p c}$. Then $\mathcal{C}$ runs PKeyGen $\left(S_{p c}, S_{I D_{j}}\right)$ and returns $S k_{a p}$ to $\mathcal{A}$.

c. Proxy Signcryption query $Q P S C\left(m, S_{I D_{j}}, I D_{u}, S k_{a p}\right): \mathcal{A}$ selects a message $m$ and three identities $I D_{i}, I D_{j}$ and $I D_{C}$. $\mathcal{C}$ first, run Extract and Proxy Credential to get the private keys of $S_{I D_{i}}, S_{I D_{j}}$ and the proxy credential $S_{p c}$, then run PKeyGen $\left(S_{p c}, S_{I D_{j}}\right)$ to get $S k_{a p}$. Finally, $\mathcal{C}$ it runs PSC $\left(m, S_{I D_{j}}, I D_{C}, S k_{a p}\right)$ and sends the result $C_{T}$ to $\mathcal{A}$.

d. Unsigncryption query $Q U S\left(C_{T}, I D_{i}, I D_{j}, I D_{C}\right): \mathcal{A}$ chooses a ciphertext $C_{T}$ and three identities $I D_{i}, I D_{j}$ and $I D_{C} \cdot \mathcal{C}$ first, run an Extract algorithm to get the $S_{I D_{C}}$. 
Then $\mathcal{C}$ runs $\mathbf{U S C}\left(C_{T}, I D_{i}, I D_{j}, S_{I D_{C}}\right)$ and sends the output to $\mathcal{A}$. This output can be the symbol $\perp$ if $C_{T}$ is an invalid ciphertext.

Challenge: $\mathcal{A}$ choose two plaintext $M_{0}, M_{1} \in M$ and two identities $I D_{B}, I D_{C}$ on which he wishes to be challenged. He cannot have asked the private key corresponding to neither $I D_{B}$ nor $I D_{C}$ in the first stage. $\mathcal{C}$ chooses a random bit $b \in\{0,1\}$ and computes $\mathcal{C}=$ signcryption $\left(M_{b}, S_{I D_{B}}, I D_{C}\right)$ that is sent to $\mathcal{A}$.

Phase 2: $\mathcal{A}$ perform again a polynomial limited number of requests like in Phase 1. Except for the Extract query on $I D_{B}$ nor $I D_{C}$ and the plaintext corresponding to $\mathcal{C}$.

Guess: $\mathcal{A}$ creates a guess a bit $b^{\prime}$ and wins the game if $b^{\prime}=b$. We define $\mathcal{A}$ 's advantage as $\operatorname{Adv}(\mathcal{A})=\left|\operatorname{Pr}\left[b^{\prime}=b\right]-\frac{1}{2}\right|$.

Definition 2. A SE-IDPSC-CS scheme is said to achieve the security requirement of EF-SE-IDPSC-CS-CMA if no polynomially time adversaries who have a non-negligible advantage in the following game:

Initial: $\mathcal{C}$ runs Setup algorithm to get params and $s$. Then sends params to $\mathcal{F}$.

The adversary $\mathcal{F}$ performs a polynomial limited number of requests just like in the gam IND-SE-IDPSC-CS-CCA2.

Finally, $\mathcal{F}$ produces a new triple $\left(C_{T}, I D_{B}, I D_{C}\right)$, where the secret key of $I D_{B}$ was not asked in the $2^{\text {nd }}$ phase and $\mathcal{F}$ wins the game if the output of Unsigncryption $\left(C_{T}, S_{I D_{B}}, I D_{C}\right)$ is not $\perp$ a symbol. The advantage of $\mathcal{F}^{\prime}$ s is simply its probability of a win.

\section{Construction}

In this section, we briefly describe the six algorithms of our SE-IDPSC-CS scheme.

1. Setup $(k)$ : on input the security parameters $k$, the PKG choose two groups $\mathbb{G}_{1}$ and $\mathbb{G}_{2}$ of prime order $q$, a generator $P$ of $\mathbb{G}_{1}$ a bilinear map $\hat{e}: \mathbb{G}_{1} \times \mathbb{G}_{1} \rightarrow \mathbb{G}_{2}$ and hash functions $H_{1}:\{0,1\}^{*} \rightarrow \mathbb{G}_{1}, H_{2}:\{0,1\}^{*} \rightarrow Z_{q^{*}}, H_{3}: \mathbb{G}_{2} \rightarrow\{0,1\}^{n}, H_{4}:\{0,1\}^{n} \times \mathbb{G}_{2} \rightarrow Z_{q^{*}}$. The PKG randomly chooses $s \in Z_{q^{*}}$ as a master key and calculates $P_{p u b}=s P$. It also chooses a secure symmetric cipher $(E, D)$. Then PKG publishes the system public parameters as params: $\left\{\mathbb{G}_{1}, \mathbb{G}_{2}, n, \hat{e}, P, P_{\text {pub }}, H_{1}, H_{2}, H_{3}, H_{4}, E, D\right\}$ and keeps the master key $s$ secret. Where $n$ is the bit length of a message.

2. Extract $(I D)$ : on input an identity $I D$, the PKG computes $Q_{I D}=H_{1}(I D)$ and $S_{I D}=s Q_{I D}$, as the public and private keys of the user's respectively and transmit the private key $S_{I D}=s Q_{I D}$ to its owner in a secure way.

3. Proxy Credential (params, $\left.S_{I D_{A}}, m_{w}\right)$ : On input params, a delegator private key $S_{I D_{A}}$, and a warrant $m_{w}$. Then, delegator generates a proxy credential $S_{p c}$ as follows:

$$
\begin{aligned}
& x \in Z_{q^{*}} \\
& U=x P \\
& z=H_{2}\left(m_{w}, U\right) \\
& S_{p c}=z S_{I D_{A}}+x P_{p u b}
\end{aligned}
$$

sends $\left(m_{w}, U, S_{p c}\right)$ to a proxy signcryptor. There is a clear explanation of the delegation privileges and some common information about original and proxy signcrypter in the $m_{w}$ which helps the receiver for verification. 
4. PKeyGen $\left(m_{w}, U, S_{p c}, S_{I D_{B}}\right)$ : Upon receiving $\left(m_{w}, U, S_{p c}\right)$, the proxy signcryptor confirms the validity of the received proxy credential by computing:

$$
\hat{e}\left(P, S_{p c}\right)=\hat{e}\left(P_{p u b}, z Q_{I D_{A}}+U\right)
$$

Here, we show the verification process for Equ (1):

$$
\begin{aligned}
\hat{e}\left(P, S_{p c}\right) & =\hat{e}\left(P_{p u b}, z Q_{I D_{A}}+U\right) \\
& =\hat{e}\left(P, z s Q_{I D_{A}}+x s P\right) \\
& =\hat{e}\left(P, z S_{I D_{A}}+x P_{p u b}\right) \\
& =\hat{e}\left(P, S_{p c}\right)
\end{aligned}
$$

If Equ (1) is true, the proxy signcryptor computes the proxy key as follows

$$
S k_{a p}=z S_{I D_{B}}+S_{p c}
$$

keep $S k_{a p}$ to itself and later it will be used to signcrypt message on behalf of the delegator.

5. Proxy Signcryption (params, $m, Q_{I D_{C}}, S k_{a p}, S_{I D_{B}}, m_{w}$ ): When the proxy signcryptor wants to signcrypt a message $m \in\{0,1\}^{n}$, on behalf of the delegator he/she first chooses $x^{\prime} \in Z_{q^{*}}$ and then computes

$$
\begin{aligned}
Q_{I D_{C}} & =H_{1}\left(I D_{C}\right) \\
k_{1} & =\hat{e}\left(P, P_{p u b}\right)^{x^{\prime}} \\
k_{2} & =H_{3}\left(\hat{e}\left(P_{p u b}, Q_{I D_{C}}\right)^{x^{\prime}}\right) \\
c & =E_{k_{2}}(m) \\
r & =H_{4}\left(c, k_{1}\right) \\
S & =x^{\prime} P_{p u b}-\left(r S_{I D_{B}}+S k_{a p}\right) \\
C_{T} & =\left(c, r, S, m_{w}\right)
\end{aligned}
$$

where $x^{\prime}$ is the random number, $E_{k_{2}}$ is the encryption function with the private key $k_{2}$. Then, the proxy signcryptor uploads the ciphertext $C_{T}=\left(c, r, S, m_{w}, U\right)$ to the cloud service provider (CSP).

6. Unsigncryption (params, $S_{I D_{C}}, C_{T}$ ): When Charlie wants the data, he can download the signcrypted ciphertext $C_{T}=\left(c, r, S, m_{w}\right)$ from a cloud service provider (CSP) and perform the following tasks:

$$
\begin{aligned}
& Q_{I D_{A}}=H_{1}\left(I D_{A}\right) \\
& Q_{I D_{B}}=H_{1}\left(I D_{B}\right) \\
& k_{1}^{\prime}=\hat{e}(P, S) \hat{e}\left(P_{p u b}, Q_{I D_{B}}\right)^{z+r} \hat{e}\left(P_{p u b}, z Q_{I D_{A}}+U\right) \\
& k_{2}^{\prime}=H_{3}\left(\hat{e}\left(S, Q_{I D_{C}}\right) \hat{e}\left(Q_{I D_{B}}, S_{I D_{C}}\right)^{z+r} \hat{e}\left(z Q_{I D_{A}}+U, S_{I D_{C}}\right)\right)
\end{aligned}
$$

then receives $m=E_{k_{2}^{\prime}}(c)$ and accepts $C_{T}$ iff $r=H_{4}\left(c, k_{1}^{\prime}\right)$. Otherwise, returns an error symbol $\perp$. 


\section{Proof of correctness}

The following equations give the correctness of our proposed scheme:

$$
\begin{aligned}
k_{1} & =\hat{e}(P, S) \hat{e}\left(P_{p u b}, Q_{I D_{B}}\right)^{z+r} \hat{e}\left(P_{p u b}, z Q_{I D_{A}}+U\right) \\
& =\hat{e}(P, S) \hat{e}\left(P_{p u b}, Q_{I D_{B}}\right)^{z+r} \hat{e}\left(P, z S_{I D_{A}}+x P_{p u b}\right) \\
& =\hat{e}(P, S) \hat{e}\left(P_{p u b}, Q_{I D_{B}}\right)^{z+r} \hat{e}\left(P, S_{p c}\right) \\
& =\hat{e}(P, S) \hat{e}\left(P, r S_{I D_{B}}\right) \hat{e}\left(P, z S_{I D_{B}}\right) \hat{e}\left(P, S_{p c}\right) \\
& =\hat{e}\left(P, x^{\prime} P_{p u b}-r S_{I D_{B}}-S k_{a p}\right) \hat{e}\left(P, r S_{I D_{B}}\right) \hat{e}\left(P, S K_{a p}\right) \\
& =\hat{e}\left(P, P_{p u b}\right)^{x^{\prime}} \\
& =k_{1} \\
k_{2} & =H_{3}\left(\hat{e}\left(S, Q_{I D_{C}}\right) \hat{e}\left(Q_{I D_{B}}, S_{I D_{C}}\right)^{z+r} \hat{e}\left(z Q_{I D_{A}}+U, S_{I D_{C}}\right)\right) \\
& =H_{3}\left(\hat{e}\left(S, Q_{I D_{C}}\right) \hat{e}\left(r S_{I D_{B}}, Q_{I D_{C}}\right)\right. \\
& \left.. \hat{e}\left(z S_{I D_{B}}+z S_{I D_{A}}+x P_{p u b}, Q_{I D_{C}}\right)\right) \\
& =H_{3}\left(\hat{e}\left(S, Q_{I D_{C}}\right) \hat{e}\left(r S_{I D_{B}}, Q_{I D_{C}}\right) \hat{e}\left(S k_{a p}, Q_{I D_{C}}\right)\right) \\
& =H_{3}\left(\hat{e}\left(x P_{p u b}-r S_{I D_{B}}-S k_{a p}, Q_{I D_{C}}\right)\right. \\
& \left.\hat{e}\left(r S_{I D_{B}}+S k_{a p}, Q_{I D_{C}}\right)\right) \\
& =H_{3}\left(\hat{e}\left(P_{p u b}, Q_{C}\right)^{x^{\prime}}\right) \\
& =k_{2}
\end{aligned}
$$

\section{Security proof}

In this section, we prove that the proposed scheme fulfills IND-SE-IDPSC-CS-CCA2 and EUF- SE-IDPSC-CS-CMA security by the following Theorems 1 and 2, respectively.

Theorem 1. (Proof of IND-SE-IDPSC-CS-CCA2 ): The proposed scheme in this paper secure against IND-SE-IDPSC-CS-CCA2, if no polynomial-time adversaries who have a non-negligible advantage $\mathcal{A}$ which can $\left(\varepsilon^{\prime}, t^{\prime}\right)$ break DBDHP where,

$$
\begin{gathered}
\varepsilon^{\prime} \geq 2\left(\varepsilon-q_{u} / 2^{k-1}\right) / q_{H_{1}}^{4} \\
t^{\prime} \approx t+O\left(q_{p k}+q_{s}+q_{u}\right) t_{\lambda}
\end{gathered}
$$

where $t_{\lambda}$ is time to calculate one pairing operation.

Proof: Assume $\mathcal{A}$ can $\left(t, q_{H_{1}}, q_{H_{2}}, q_{H_{3}}, q_{H_{4}}, q_{e}, q_{p k}, q_{s}, q_{u}, \varepsilon\right)$ - break the SE-IDPSC-CS scheme with significant advantage $\varepsilon$ under adaptive CCA2 after running $t$ (time) and requesting at most $q_{H_{i}}$ random oracle for ( $i=1$ to 4$), q_{e}$ Extract query, $q_{p k}$ PKeyGen query, $q_{s}$ proxy signcryption query, and $q_{u}$ unsigncryption query. Then we can build another algorithm $\mathcal{C}$ that $\left(t^{\prime}, \varepsilon^{\prime}\right)$-breaks the DBDHP by taking $\mathcal{A}$ as a subroutine.

Assume $\mathcal{C}$ obtains a random instance $(P, a P, b P, c P, h)$ of the DBDHP and the objective of $\mathcal{C}$ is to obtain $h=\hat{e}(P, P)^{a b c}$ or not. 
Initial: In this proof, $\mathcal{C}$ pretends $\mathcal{A}$ 's challenger in the IND-SE-IDPSC-CS-CCA2 game.

Phase 1: $\mathcal{C}$ maintains five empty lists $L_{1}, L_{2}, L_{3}, L_{4}$ and $L_{a p}$ which used to simulate the hash oracles $H_{1}, H_{2}, H_{3}$ and $H_{4}$ respectively and $L_{a p}$ is an empty list which is used to describe the Extract and PKeyGen query Oracles and $L_{4}$ will be used to simulate the proxy signcryption query.

At the beginning of the game, $\mathcal{C}$ gives $\mathcal{A}$ params with $P_{\text {pub }}=c P$. Then, $\mathcal{C}$ chooses two distinct random numbers $i, j \in\left\{1, \ldots, q_{H_{1}}\right\}$. $\mathcal{A}$ requests a number of $H_{1}$ queries on identities of his/her choice. At $i^{\text {th }} H_{1}$ request, $\mathcal{C}$ answers $H_{1}\left(I D_{i}\right)=a P$. At $j^{\text {th }} H_{1}$ request, $\mathcal{C}$ answers $H_{1}\left(I D_{j}\right)=b P$. Since, $a P$ and $b P$ belong to DBDHP, $\mathcal{A}$ 's understanding will not be changed by these variations. Hence, the private keys $S_{I D_{i}}$ and $S_{I D_{j}}$ are $a c P$ and $b c P$ respectively. Thus the solution $\hat{e}(P, P)^{a b c}$ of the DBDHP is given by $\hat{e}\left(Q_{I D_{i}}, S_{I D_{j}}\right)=\left(S_{I D_{i}}, Q_{I D_{j}}\right)$. For requests $H_{1}\left(I D_{e}\right)$ with $e \neq i, j, \mathcal{C}$ chooses $b_{e} \leftarrow Z_{q^{*}}$, puts the pair $\left(I D_{e}, b_{e}\right)$ in list $L_{1}$ and answers $H_{1}\left(I D_{e}\right)=b_{e} P$.

Let see how $\mathcal{A}$ issues the other kinds of request.

1. $H_{2}$ requests: $\mathcal{A}$ sends a query to $\mathrm{H}_{2}$ oracle $\mathcal{C}$ checks $L_{2}$ for a matched entry. If similar entry is found in $L_{2}, \mathcal{C}$ returns $z$. Otherwise, $\mathcal{C}$ randomly chooses $z \in Z_{q^{*}}$ and stores the entry $\left(m_{w}, U, z\right)$ into $L_{2}$.

2. $H_{3}$ requests: on $H_{3}\left(g_{e}\right)$ request, $\mathcal{C}$ searches $\left(g_{e}, R_{e}\right)$ in the list $L_{3}$. If similar entry is found in $L_{3}, \mathcal{C}$ replies $R_{e}$ otherwise $\mathcal{C}$ answer $\mathcal{A}, R_{e} \leftarrow\{0,1\}^{n}$ such that, no entry $(., R)$ exists in $L_{3}$ and puts the pair $\left(g_{e}, R\right)$ into $L_{3}$.

3. $H_{4}$ requests: for $H_{4}\left(c_{e}, k_{e}\right)$ query, $\mathcal{C}$ first checks $L_{4}$ does not contain a tuple $\left(c_{e}, k_{e}, r_{e}\right)$. If $\left(c_{e}, k_{e}, r_{e}\right)$ is found, $\mathcal{C}$ answers $r_{e}$, otherwise $\mathcal{C}$ choose $r \leftarrow Z_{q^{*}}$ and give it the query as an answer and place $\left(c_{e}, k_{e}, r\right)$ into $L_{4}$.

4. Extract query: when $\mathcal{A}$ asks $\operatorname{Extract}\left(I D_{A}\right)$, then $\mathcal{C}$ fails and stops, if $I D_{A}=I D_{i}$ or $I D_{A}=I D_{j}$. If $I D_{A} \neq I D_{i}, I D_{j}$ then $L_{1}$ must contain a pair $\left(I D_{A}, d\right)$ for some $d$ (It shows $\mathcal{C}$ previously answered $H_{1}\left(I D_{A}\right)=d P H_{1}$ query on $\left.I D_{A}\right)$. Then $\mathcal{C}$ computes $d P_{\text {pub }}=c d P$ as $I D_{A}$ private key and returned to $\mathcal{A}$.

5. PKeyGen query: Let us assume $\mathcal{A}$ has made the $\mathrm{H}_{2}$ and extraction query before the proxy key query. When $\mathcal{A}$ makes a proxy key query of the tuple $\left(I D_{A}, I D_{B}, m_{w}\right)$ for the proxy signcryptor, $\mathcal{C}$ first check the $L_{2}$ list for a matching entry. Otherwise, $\mathcal{C}$ randomly choose $x_{a} \in Z_{q^{*}}$ to compute.

$$
\begin{aligned}
& x_{a} \in Z_{q^{*}} \\
& U_{a}=x_{a} P \\
& z=H_{2}\left(m_{w}, U_{a}\right) \\
& S_{p c}=z S_{I D_{A}}+x_{a} P_{p u b}
\end{aligned}
$$

If $\hat{e}\left(P, S_{p c}\right)=\hat{e}\left(P_{p u b}, z Q_{I D_{A}}+U_{a}\right)$ holds $\mathcal{C}$ compute the proxy key $S k_{a p}$ using the equality $S k_{a p}=z S_{I D_{B}}+S_{p c}$ and return $S k_{a p}$ to the adversary. Finally, $\mathcal{C}$ stores $\left(S k_{a p}, z, m_{w}\right)$ to $L_{a p}$.

6. Proxy Signcryption query: Assume $\mathcal{A}$ has made many Hash, Extract, PKeyGen queries before $q_{s}$ query. $\mathcal{A}$ request a query of $\left(M, I D_{B}, I D_{C}, m_{w}, S K_{a p}\right)$ to Proxy Signcryption query. $\mathcal{C}$ considers the following two cases as a reply.

Case 1: If $I D_{B}$ and $I D_{C}$ are not the identities $I D_{i}$ and $I D_{j}$. In the case $I D_{B} \neq I D_{i}, I D_{j}, \mathcal{C}$ run an Extract algorithm to calculate $S_{I D_{B}}$ of $I D_{B}$ and then can 
simply run Signcryption $\left(m, S_{I D_{B}}, Q_{I D_{C}}\right)$ and send $C_{T}$ to $\mathcal{A}$.

Case 2: If $I D_{B}=I D_{i}$ or $I D_{B}=I D_{j}$ and $I D_{C} \neq I D_{i}, I D_{j}, \mathcal{C}$ has to simulate the execution of the Proxy Signcryption query as follows. $\mathcal{C}$ chooses $r, z \in Z_{q^{*}}$ and $S \in \mathbb{G}_{1}^{*}$. Then, computes:

$$
\begin{aligned}
k^{\prime} & =\hat{e}(P, S) \hat{e}\left(P_{p u b}, Q_{I D_{B}}\right)^{z+r} \hat{e}\left(P_{p u b}, z Q_{I D_{A}}+U\right) \text { and } \\
\tau & =\hat{e}\left(S, Q_{I D_{C}}\right) \hat{e}\left(Q_{I D_{B}}, S_{I D_{C}}\right)^{z+r} \hat{e}\left(z Q_{I D_{A}}+U, S_{I D_{C}}\right)
\end{aligned}
$$

where $S_{I D_{C}}$ is the private key of $I D_{C} . \mathcal{C}$ runs $H_{3}$ algorithm to find $k_{2}=H_{3}(\tau)$ and calculates $c=E_{k_{2}}(M)$. $\mathcal{C}$ checks if $L_{4}$ contains $\left(c, k^{\prime}, r^{\prime}\right)$ with $r^{\prime} \neq r$. $\mathcal{C}$ repeats checking with another pair $(r, S)$ until he found $\left(c, k^{\prime}, r\right)$. Before obtaining an allowable $\left(k^{\prime}, r, S\right), \mathcal{C}$ must repeat the checking at most $2 q_{R}$ times. At each attempt, $\mathcal{C}$ must calculate four pairings $\hat{e}$. Once $\left(k^{\prime}, r, S\right)$ is found, $\mathcal{C}$ puts $\left(c, k^{\prime}, r\right)$ into $L_{4}$ before returning $(c, r, S)$ which will look to be effective from $\mathcal{A}$ 's point of view.

If $I D_{B}$ and $I D_{C}$ are the identities of $I D_{i}$ and $I D_{j}, \mathcal{C}$ signcrypt $M$ like this. $\mathcal{C}$ chooses $r^{*} \in Z_{q^{*}}$ and $S^{*} \in \mathbb{G}_{1}$ computes $k_{1}^{\prime}=\hat{e}\left(P, S^{*}\right) \hat{e}\left(P_{p u b}, Q_{I D_{b}}\right)^{r^{*}}=\hat{e}\left(P, S^{*}\right) \hat{e}(c P, b P)^{r^{*}}$ and chooses random $\tau^{*} \in \mathbb{G}_{2}$ and $k_{2}^{\prime} \in\{0,1\}^{n}$ such that no entry $\left(., k_{2}^{\prime}\right)$ is in $L_{2}$ and computes $c^{*}=E_{k_{2}^{\prime}}(M)$. $\mathcal{C}$ then confirms if $L_{3}$ contains $\left(c^{*}, k_{1}^{\prime}, r^{*}\right)$ such that $r^{\prime} \neq r^{*}$. If not, $\mathcal{C}$ puts the $\left(c^{*}, k_{1}^{\prime}, r^{*}\right)$ into $L_{4}$ and $\left(\tau^{*}, k_{2}^{\prime}\right)$ into $L_{3}$. In the opposite case, $\mathcal{C}$ chooses another random pair $\left(r^{*}, S^{*}\right)$ and repeats the above process until $\mathcal{C}$ finds a tuple $\left(c^{*}, k_{1}^{\prime}, r^{*}\right)$. Once $\mathcal{C}$ has admissible elements $\left(r^{*}, S^{*}\right), \mathcal{C}$ gives the ciphertext $C_{T}^{*}=\left(c^{*}, r^{*}, S^{*}\right)$ to $\mathcal{A}$. $\mathcal{A}$ will never know that $C_{T}^{*}$ is not a valid ciphertext of $M$ for $I D_{i}$ and $I D_{j}$ since $\mathcal{C}$ will not ask unsigncrypt of $C_{T}^{*}$.

Unsigncrypt query: Once $\mathcal{A}$ observes $C_{T}^{*}=\left(c^{*}, r^{*}, S^{*}\right)$ for $I D_{B}$ and $I D_{C}, \mathcal{A}$ needs to ask $\mathcal{C}$ for unsigncryption $C_{T}^{*}$. In this, case $\mathcal{C}$ informs $\mathcal{A}$ that $C_{T}^{*}$ is invalid: if $\mathcal{A}$ before requesting the hash value $H_{3}\left(c^{\prime}, \hat{e}(P, S) \hat{e}\left(P_{p u b}, Q_{I D_{B}}\right)^{z+r} \hat{e}\left(P_{p u b}, z Q_{I D_{A}}+U\right), \mathcal{C}\right.$ has a probability of at most $1 / 2^{k}$ to answer $r^{\prime}$. This will fails if $L_{4}$ contains $\left(c^{\prime}, \hat{e}(P, S) \hat{e}\left(P_{p u b}, Q_{I D_{B}}\right)^{z+r}\right.$ $\left.\hat{e}\left(P_{p u b}, z Q_{I D_{A}}+U\right), r^{\prime}\right)$. When getting an Unsigncrypt query for $C_{T}^{*}=\left(c^{*}, r^{*}, S^{*}\right)$ identities $I D_{B}$ and $I D_{C}$ that is not $I D_{i}$ and $I D_{j}, \mathcal{C}$ first calculates $k_{1}^{\prime}=\hat{e}\left(P, S^{\prime}\right) \hat{e}\left(P_{p u b}, Q_{I D_{B}}\right)^{z+r^{\prime}} \hat{e}\left(P_{p u b}, z Q_{I D_{A}}+U\right)$ and checks if the list $L_{4}$ contains the tuple $\left(c^{\prime}, k_{1}^{\prime}, r^{\prime}\right)$. If it is not found, $\mathcal{C}$ reject the ciphertext. Otherwise, $\mathcal{C}$ can recover $r^{\prime}$ and calculate $\tau^{\prime}=\hat{e}\left(S^{\prime}, Q_{I D_{C}}\right) \hat{e}\left(Q_{I D_{B}}, S_{I D_{C}}\right)^{z+r^{\prime}} \hat{e}\left(z Q_{I D_{A}}+U, S_{I D_{C}}\right) . \mathcal{C}$ then searches for $H_{3}\left(\tau^{\prime}\right)$ queries in the list $L_{3}$. If $H_{3}\left(\tau^{\prime}\right)$ query is not found in $L_{3}, \mathcal{C}$ takes $\left(\tau, k_{2}^{\prime}\right) \in \mathbb{G}_{2} \times\{0,1\}^{n}$ such that no $\left(., k_{2}^{\prime}\right)$ already exists in $L_{3}$ and inserts $\left(\tau, k_{2}^{\prime}\right)$ into $L_{3} . \mathcal{C}$ finally uses $k_{2}^{\prime}$ to find $m^{\prime}=D_{k_{2}^{\prime}}\left(c^{\prime}\right)$ and returns $m^{\prime}$. Here we can easily observe that the probability of refusing a valid ciphertext does not exceed $q u / 2^{k}$. Definitely, for a query on identities $I D_{B}$ and $I D_{C}$ that is not $I D_{i}$ and $I D_{j}$, if $\mathcal{A}$ later requests $H_{3}\left(c^{\prime}, \hat{e}\left(P, S^{\prime}\right) \hat{e}\left(P_{p u b}, Q_{I D_{B}}\right)^{z+r^{\prime}} \hat{e}\left(P_{p u b}, z Q_{I D_{A}}+U\right)\right)$, there is a probability of at most $1 / 2^{k}$ that $\mathcal{C}$ answers $r^{\prime}$. After a polynomial limited number of queries $\mathcal{A}$ chooses $\left(I D_{i}, I D_{j}\right)$ which he needs to be challenged with a probability at least $\left(1 / 2^{q H_{1}}\right)$. Notice that, if $\mathcal{A}$ actually selects to be challenged on $I D_{i}$ and $I D_{j}$, then in the $2^{\text {nd }}$ stage $\mathcal{A}$ cannot ask the private key of $I D_{i}$ and $I D_{j}$. If $\mathcal{A}$ does not select $I D_{i}$ and $I D_{j}$ as target identities, then $\mathcal{C}$ fails. When $\mathcal{A}$ 
produces two plaintexts $m_{0}$ and $m_{1}, \mathcal{C}$ selects a random bit $b \in\{0,1\}$ and signcrypt $m_{b}$. To do so $\mathcal{C}$ chooses $r^{*} \leftarrow Z_{q^{*}}$ and $S^{*} \leftarrow \mathbb{G}_{1}$.

$\mathcal{C}$ calculates $k_{1}^{\prime}=\hat{e}\left(P, S^{*}\right) \hat{e}\left(P_{p u b}, Q_{I D_{b}}\right)^{r^{*}}=\hat{e}\left(P, S^{*}\right) \hat{e}(c P, b P)^{r^{*}}, \tau^{*}=\hat{e}\left(S^{*}, Q_{I D_{C}}\right)^{h^{r^{*}}}$

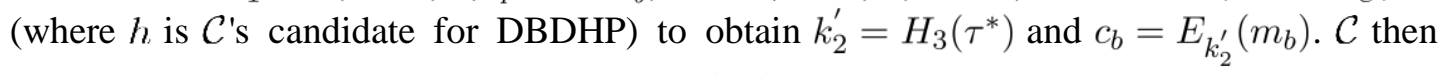
verifies if $L_{4}$ already contains an entry $\left(c_{b}, k_{1}^{\prime}, r^{\prime}\right)$ such that $r^{\prime} \neq r^{*}$. If not, $\mathcal{C}$ puts the tuple $\left(c_{b}, k_{1}^{\prime}, r^{*}\right)$ into $L_{4}$. In the reverse case, $\mathcal{C}$ chooses another random pair $\left(r^{*}, S^{*}\right)$ and repeats the procedure until finding a tuple $\left(c_{b}, k_{1}^{\prime}, r^{*}\right)$. Once $\mathcal{C}$ has admissible elements $\left(r^{*}, S^{*}\right), \mathcal{C}$ just have to send the ciphertext $\sigma=\left(c_{b}, r^{*}, S^{*}\right)$ to $\mathcal{A}$.

$\mathcal{A}$ then runs the second queries which are similar to the above one, finally $\mathcal{A}$ produces a bit $b^{\prime}$ for the relation $C_{T}=$ Signcrypt $\left(m_{b^{\prime}}, S_{I D_{i}}, I D_{j}\right)$ holds. At this moment, if $b=b^{\prime}, \mathcal{C}$ answers 1 because his candidate $h$ permitted him to create $\sigma$ that seemed to $\mathcal{A}$ as a valid signcrypted message of $m_{b}$. If $b \neq b^{\prime}, \mathcal{C}$ then answers 0 . We must have to calculate $\mathcal{C}$ 's probability of success. We saw that $\mathcal{C}$ fails if $\mathcal{A}$ asks the private key of either $I D_{i}$ or $I D_{j}$ in the first phase, and also we know that there are $\left(2^{q H_{1}}\right)$ ways to select $I D_{i}, I D_{j}$ among those $\left(2^{q H_{1}}\right)$ pairs of identities, none of them are the subject of an Extract query from $\mathcal{A}$. Then, with a probability greater than $1 /\left(2^{q H_{1}}\right), \mathcal{A}$ will not ask Extract $\left(I D_{i}\right)$ and Extract $\left(I D_{i}\right)$. Additionally, with a probability exactly $1 /\left(2^{q H_{1}}\right), \mathcal{A}$ selects to be challenged on the pair $I D_{i}, I D_{j}$ and this must allow $\mathcal{C}$ solving his decisional problem if $\mathcal{A}$ wins the IND-SE-IDPSC-CS-CCA2 game.

Finally,

$$
\begin{aligned}
& p_{1}=P\left[b^{\prime}=b \mid C_{T}=\operatorname{Signcrypt}\left(m_{b}, S_{I D_{i}}, I D_{j}\right)\right] \\
& =\frac{\varepsilon+1}{2}-\frac{q U}{2^{k}}, \text { and } \\
& p_{0}=P\left[b^{\prime}=i \mid h \in \mathbb{G}_{2}\right]=\frac{1}{2}, \text { for } i=0,1, \text { we then have } \\
& A d v(\mathcal{C})=\mid P_{a, b, c} \in Z_{q^{*}}\left[1 \leftarrow \mathcal{C}\left(a P, b P, c P, \hat{e}(P, P)^{a b c}\right)\right]- \\
& P_{a, b, c} \in Z_{q^{*}}, h \in \mathbb{G}_{2}[1 \leftarrow \mathcal{C}(a P, b P, c P, h)] \mid \\
& =\frac{\left|p_{1}-p_{0}\right|}{\left(2^{q H_{1}}\right)^{2}}=\frac{\varepsilon-q u / 2^{k-1}}{2\left(2^{q H^{1}}\right)^{2}}>\frac{2\left(\varepsilon-q u / 2^{k-1}\right)}{q H_{1}^{4}} .
\end{aligned}
$$

We note that the denominator is $q H_{1}^{4}$ rather than $q H_{1}^{2}$

Theorem 2. (Proof of EUF-SE-IDPSC-CS-CMA): The proposed scheme in this paper secure against EUF-SE-IDPSC-CS-CMA security if no probabilistic polynomial-time adversary $\mathcal{F}$ with a non-negligible advantage that can $\left(\varepsilon^{\prime}, t^{\prime}\right)$ break CDHP where,

$$
\begin{aligned}
& \varepsilon \geq 10\left(q_{s}+1\right)\left(q_{s}+q_{H_{3}}\right) q_{H_{1}} /\left(2^{k}-1\right) \\
& t^{\prime} \leq 120686 q_{H_{1}} q_{H_{3}} \frac{t+O\left(\left(q_{p k}+q_{s}+q_{u} q_{H_{3}}\right) t_{\lambda}\right)}{\hat{e}\left(1-1 / 2^{k}\right)}
\end{aligned}
$$

where $t_{\lambda}$ is time to calculate one pairing operation. 
Proof: Assume $\mathcal{A}$ can $\left(t, q_{H_{1}}, q_{H_{2}}, q_{H_{3}}, q_{H_{4}}, q_{e}, q_{p k}, q_{s}, \varepsilon\right)$-break our SE-IDPSC-CS scheme with non-negligible advantage $\varepsilon$ under the adaptive CMA after running in $t$ (time) and asking at most $q_{H_{i}}$ random oracle for ( $i=1$ to 4 ), $q_{e}$ extraction query, $q_{p k}$ PKeyGen query, $q_{s}$ proxy signcryption query. Then we can build another algorithm $\mathcal{C}$ that $\left(t^{\prime}, \varepsilon^{\prime}\right)$-breaks the CDHP by taking $\mathcal{F}$ as a subroutine. Assume the algorithm $\mathcal{C}$ takes as the input $(P, a P, b P)$ of the CDHP, and the objective of $\mathcal{C}$ is to compute $a b P$.

As proof in [18], this theorem using the famous forking lemma [31]. $\mathcal{C}$ simulates $\mathcal{F}$ 's a challenger in the EUF-SE-IDPSC-CS-CMA game. $\mathcal{F}$ adaptively asks queries as described in the EUF-SE-IDPSC-CS-CMA game. We define the procedure as follows.

Initial: $\mathcal{C}$ runs Setup algorithm with $k$ and gives $\mathcal{F} P_{p u b}=b P$.

Attack: $\mathcal{C}$ simulates $\mathcal{F}$ 's a challenger in the EUF-SE-IDPSC-CS-CMA game and answers $\mathcal{F}$ 's queries according to the procedures in Theorem 1. In addition, we need to set the challenge identity $I D_{A}=I D_{l}$ in $H_{1}$ queries.

Forgery: $\mathcal{F}$ outputs a triple $\left(I D_{A}, I D_{B}, C_{T}\right)$, where $C_{T}=(X, y, \tau)$ (note that $\mathcal{C}$ can extract message-signature pair from $C_{T}$ since it identifies $S_{I D_{B}}$ of $I D_{B}$ because of irreflexivity assumption). Since the dividing lemma is only suitable for identity-less chosen message attacks, we need to merge the message $m$ and the sender identity $I D_{A}$ into a "general" forged message $\left(I D_{A}, m\right)$. From the dividing lemma, if $\mathcal{F}$ is an effective forger in the above game, we can build a Las Vegas machine $\mathcal{F}^{\prime}$ that outputs $\left(\left(I D_{A}, m\right), h, Z\right)$ and $\left(\left(I D_{A}, m\right), h^{*}, Z^{*}\right)$ with $h \neq h^{*}$ and the same commitment. To solve the given CDHP based on the machine $\mathcal{F}^{\prime}$ derived from $\mathcal{F}$, we build $\mathcal{C}$ as follows.

1) $\mathcal{C}$ obtains two different signatures $\left(\left(I D_{A}, m\right), h, Z\right)$ and $\left(\left(I D_{A}, m\right), h^{*}, Z^{*}\right)$ by running $\mathcal{F}^{\prime}$.

2) $\mathcal{C}$ computes $a b P=\left(h-h^{*}\right)^{-1}\left(Z-Z^{*}\right)$.

3) $\mathcal{C}$ computes $a b P$ as the solution to the $\mathrm{CDH}$ problem.

From the dividing lemma and the lemma on the affiliation among the chosen identity attack and the given-identity attack [35], if $\mathcal{F}$ flourishes in time $t$ with probability $\varepsilon \geq 10\left(q_{s}+1\right)\left(q_{s}+q_{H_{3}}\right) q_{H_{1}} /\left(2^{k}-1\right)$, then $\mathcal{C}$ can solve the given CDHP in the expected time

$$
t^{\prime} \leq 120686 q_{H_{1}} q_{H_{3}} \frac{t+O\left(\left(q_{p k}+q_{s}+q_{u} q_{H_{3}}\right) t_{\lambda}\right)}{\hat{e}\left(1-1 / 2^{k}\right)}
$$

Table 2. Shows the comparison of computational cost and security

\begin{tabular}{|c|c|c||c||c||c|c||}
\hline & & & \multicolumn{2}{c|}{ Security } \\
\cline { 6 - 7 } Schemes & PKeyGen & Proxy Signcryption & Unsigncryption & Total & IND-CCA2 & EUF-CMA \\
\hline$[22]$ & $3 \mathrm{M}+2 \mathrm{P}+1 \mathrm{E}$ & $3 \mathrm{M}+2 \mathrm{P}+2 \mathrm{E}$ & $1 \mathrm{M}+5 \mathrm{P}+1 \mathrm{E}$ & $7 \mathrm{M}+9 \mathrm{P}+4 \mathrm{E}$ & $\checkmark$ & $\checkmark$ \\
\hline \hline$[23]$ & $2 \mathrm{M}+2 \mathrm{P}+1 \mathrm{E}$ & $1 \mathrm{M}+2 \mathrm{P}+2 \mathrm{E}$ & $4 \mathrm{E}+6 \mathrm{P}$ & $3 \mathrm{M}+10 \mathrm{P}+7 \mathrm{E}$ & $\checkmark$ & $\checkmark$ \\
\hline \hline$[25]$ & $2 \mathrm{M}+2 \mathrm{P}+1 \mathrm{E}$ & $3 \mathrm{M}+1 \mathrm{P}+1 \mathrm{E}$ & $1 \mathrm{M}+4 \mathrm{P}+1 \mathrm{E}$ & $6 \mathrm{M}+7 \mathrm{P}+3 \mathrm{E}$ & $\checkmark$ & $\checkmark$ \\
\hline \hline Ours & $2 \mathrm{M}+2 \mathrm{P}$ & $2 \mathrm{M}$ & $2 \mathrm{M}+4 \mathrm{P}$ & $6 \mathrm{M}+6 \mathrm{P}$ & $\checkmark$ & $\checkmark$ \\
\hline
\end{tabular}




\section{Performance analysis}

Following the idea of [20-21], the pairing $\hat{e}\left(P, P_{\text {pub }}\right)$ is pre-computed and when users frequently talk with each other all $\hat{e}\left(P_{p u b}, Q_{I D_{A}}\right), \hat{e}\left(P_{p u b}, Q_{I D_{B}}\right), \hat{e}\left(P_{p u b}, Q_{I D_{C}}\right)$, $\hat{e}\left(Q_{I D_{A}}, S_{I D_{C}}\right)$ and $\hat{e}\left(Q_{I D_{B}}, S_{I D_{C}}\right)$ can be pre-computed by the sender and receiver once for all.

\subsection{Comparison}

In this section, we compare the performance of our scheme with related schemes such as

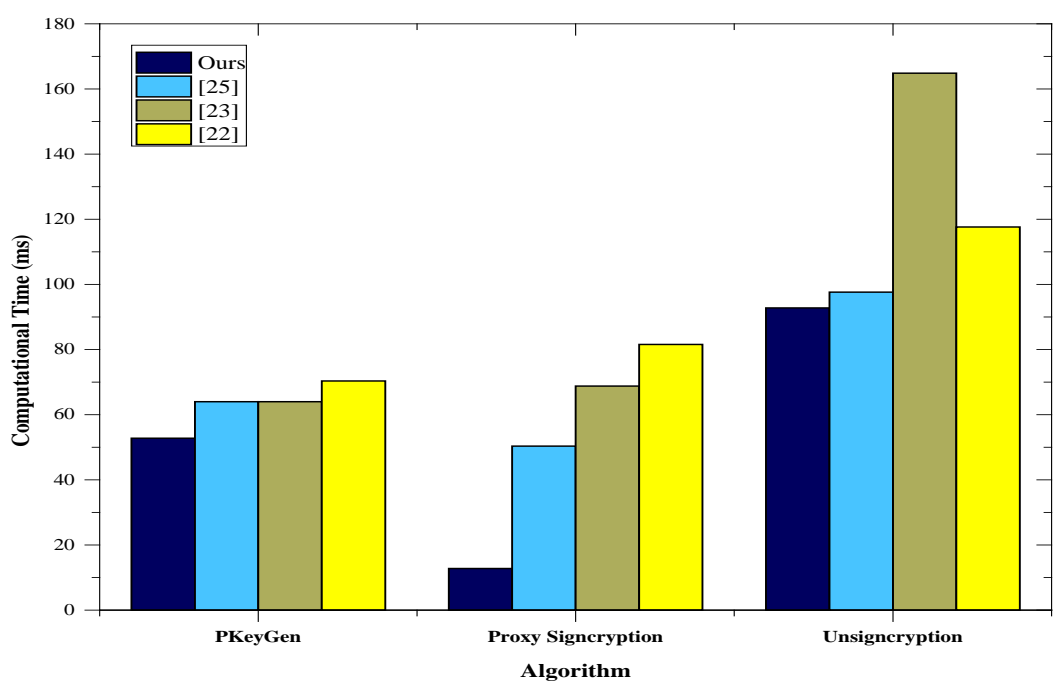

Fig. 2. Computational time of our scheme

Chen et al. [22], Ming et al. [23] and $\mathrm{H} \mathrm{Yu} \mathrm{[25]} \mathrm{interims} \mathrm{of} \mathrm{efficiency} \mathrm{and} \mathrm{security.} \mathrm{We} \mathrm{denote}$ $M$ one scalar multiplication operation in $\mathbb{G}_{1}, E$ exponentiation computation and $P$ the pairing operation in $\mathbb{G}_{2}$. In Table 2 , a symbol $\sqrt{ }$ means that all the schemes satisfy the related security requirement. Our experiment was implemented on the hardware platform of ASUS Z-Book with an Intel (R) Core ${ }^{\mathrm{TM}} \mathrm{i} 3-6100 \mathrm{U}$ CPU $2.3 \mathrm{GHz}$ and 4 GB memory running 64-bit Windows 10 operating system. According to Cao [38], time spent on one scalar multiplication operation in $\mathbb{G}_{1}$ is approximately $6.38 \mathrm{~ms}$, one exponentiation computation in $\mathbb{G}_{2}$ and paring are approximately $11.20 \mathrm{~ms}$ and $20.01 \mathrm{~ms}$ respectively. From the comparison result shown in Table 2, one can see that the computation cost of our SE-IDPSC-CS scheme is lower than other schemes and Fig. 2 clearly shows that our SE-IDPSC-CS scheme is much more efficient than the present schemes. It is known from Table 2 that all the schemes satisfy the IND-CCA2 and EUF-CMA security requirements. 


\section{Conclusion}

In this paper, we explain a new secure and efficient identity-based proxy signcryption in cloud data sharing (SE-IDPSC-CS) which is secure and efficient than the current schemes. The idea behind our proposed scheme is as follow, the manager of the company that is the original signcryptor officially delegate his/ her signcryption authority to the proxy signcryptor, then the proxy signcrypter act as a manager and generate a signcrypted messages on his/her behalf and upload the signcrypted ciphertext to cloud service provider (CSP) it is a trusted server which supplies storage services and sends the signcrypted ciphertexts to an authorized users. Finally, an authorized user download, decrypt and confirm the source and validity of the message. We also prove the security of the scheme in terms of IND-SE-IDPSC-CS-CCA2 and EF-SE-IDPSC-CS-CMA under DBDH and CDH problems respectively. Finally, we will work to design an outsourced ID-based proxy signcryption scheme in cloud data sharing as our future work to reduce the signing computational overload both in the delegate and user side.

\section{Acknowledgments}

This work was supported in part by the 13th Five-Year Plan of National Cryptography Development Fund for Cryptographic Theory of China under Grant MMJJ20170204, in part by the Fundamental Research Funds for the Central Universities under Grant ZYGX2016J091, the Guangxi Colleges and Universities Key Laboratory of Cloud Computing and Complex Systems, and in part by the Natural Science Foundation of China under Grants U1401257, 61472064 and 61602096.

\section{References}

[1] Y. Zheng, "Digital Signcryption or how to achieve cost (signature \& encryption) $<<$ cost (signature)+ cost (encryption)," in Proc. of Advances in Cryptology - CRYPTO '97, pp 165-179, 1997. Article (CrossRef Link)

[2] F. Li, B. Liu, and J. Hong, "An efficient signcryption for data access control in cloud computing," Computing, vol. 99, no. 5, pp. 465-479, 2017. Article (CrossRef Link).

[3] R.-J. Hwang, C.-H. Lai, and F.-F. Su, "An efficient signcryption scheme with forward secrecy based on elliptic curve," Applied Mathematics and computation, vol. 167, no. 2, pp. 870-881, 2005. Article (CrossRef Link).

[4] H. Y. Jung, D. H. Lee, J. I. Lim, and K. S. Chang, "Signcryption schemes with forward secrecy," in Proc. of WISA2001, Springer-Verlag, pp. 4303-475, 2001.

[5] Y. Zheng and H. Imai, "How to construct efficient signcryption schemes on elliptic curves," Information Processing Letters, vol. 68, no. 5, pp. 227-233, 1998. Article (CrossRef Link).

[6] C. Gamage, J. Leiwo, and Y. Zheng, "An efficient scheme for secure message transmission using proxy-signcryption," in Proc. of the 22nd Australasian Computer Science Conference, Springer, pp. 420-431, 1999.

[7] C. Zhou, Z. Zhao, W. Zhou, and Y. Mei, "Certificateless key-insulated generalized signcryption scheme without bilinear pairings," Security and Communication Networks, vol. 2017, 17 pages, 2017. Article (CrossRef Link).

[8] V. Saraswat, R. A. Sahu, and A. K. Awasthi, "A secure anonymous proxy signcryption scheme," Journal of Mathematical Cryptology, vol. 11, no. 2, pp. 63-84, 2017. Article (CrossRef Link).

[9] P. Pandiaraja, P. Vijayakumar, V. Vijayakumar, and R. Seshadhri, "Computation efficient attribute based broadcast group key management for secure document access in public cloud.” J. Inf. Sci. Eng., vol. 33, no. 3, pp. 695-712, 2017. Article (CrossRef Link). 
[10] A. Shamir, "Identity-based cryptosystems and signature schemes," in Proc. of Workshop on the theory and application of cryptographic techniques, Springer, pp. 47-53, 1984. Article (CrossRef Link).

[11] J. Malone-Lee, “Identity-based signcryption.” IACR Cryptology ePrint Archive, vol. 2002, p. 98, 2002.

[12] J. Xie, Y.-p. Hu, J.-t. Gao, and W. Gao, "Efficient identity-based signature over ntru lattice," Frontiers of Information Technology \& Electronic Engineering, vol. 17, no. 2, pp. 135-142, 2016. Article (CrossRef Link).

[13] Z. Qin, C. Yuan, Y. Wang, and H. Xiong, "On the security of two identity-based signature schemes based on pairings," Information Processing Letters, vol. 116, no. 6, pp. 416-418, 2016. Article (CrossRef Link).

[14] X. Hu, H. Xu, J. Wang, W. Tan, and Y. Yang, "A generic construction of identity-based proxy signature scheme in the standard model," International Journal of Information and Computer Security, vol. 11, no. 1, pp. 83-100, 2019. Article (CrossRef Link).

[15] P. S. Barreto, B. Libert, N. McCullagh, and J.-J. Quisquater, "Efficient and provably-secure identity-based signatures and signcryption from bilinear maps," in Proc. of International conference on the theory and application of cryptology and information security, Springer, pp. 515-532, 2005. Article (CrossRef Link).

[16] A. Karati, S. H. Islam, G. Biswas, M. Z. A. Bhuiyan, P. Vijayakumar, and M. Karuppiah, "Provably secure identity-based signcryption scheme for crowdsourced industrial Internet of things environments," IEEE Internet of Things Journal, vol. 5, no. 4, pp. 2904-2914, 2018. Article (CrossRef Link).

[17] X. Zhang, C. Xu, and J. Xue, "Efficient multi-receiver identity-based signcryption from lattice assumption," International Journal of Electronic Security and Digital Forensics, vol. 10, no. 1, pp. 20-38, 2018. Article (CrossRef Link).

[18] L. Chen and J. Malone-Lee, "Improved identity-based signcryption," in Proc. of International Workshop on Public Key Cryptography, Springer, pp. 362-379, 2005. Article (CrossRef Link).

[19] S. S. Chow, S.-M. Yiu, L. C. Hui, and K. Chow, "Efficient forward and provably secure id-based signcryption scheme with public verifiability and public ciphertext authenticity," in Proc. of International Conference on Information Security and Cryptology, Springer, pp. 352-369, 2003. Article (CrossRef Link).

[20] B. Libert and J.-J. Quisquater, “A new identity based signcryption scheme from pairings,” in Proc. of Information Theory Workshop, pp. 155-158, 2003. Article (CrossRef Link).

[21] X. Li and K. Chen, "Identity based proxy-signcryption scheme from pairings," in Proc. of Services Computing, 2004.(SCC 2004). Proceedings. 2004 IEEE International Conference on. IEEE, 2004, pp. 494-497, 2004. Article (CrossRef Link)

[22] S.-X. Chen, S.-X. Zhou, X.-F. Yao, and F.-W. Li, "Efficient identity-based proxy signcryption scheme,” Application Research of Computers, vol. 7, p. 084, 2011.

[23] Y. Ming, J. Feng, and J. Hu Q, "Secure identity-based proxy signcryption scheme in standard model,” Journal of Computer Applications, vol. 34, no. 10, pp. 2834-2839, 2014.

[24] C.-X. Zhou, "Identity-based generalized proxy signcryption scheme," Information Technology and Control, vol. 45, no. 1, pp. 13-26, 2016. Article (CrossRef Link).

[25] H. Yu, Z. Wang, J. Li, and X. Gao, "Identity-based proxy signcryption protocol with universal composability," Security and Communication Networks, vol. 2018, 11 pages, 2018. Article (CrossRef Link).

[26] M. Mambo, K. Usuda, and E. Okamoto, "Proxy signatures: Delegation of the power to sign messages," IEICE transactions on fundamentals of electronics, communications and computer sciences, vol. 79, no. 9, pp. 1338-1354, 1996.

[27] S. Kim, S. Park, and D. Won, "Proxy signatures, revisited," in Proc. of International Conference on Information and Communications Security, Springer, pp. 223-232, 1997. Article (CrossRef Link).

[28] B. Lee, H. Kim, and K. Kim, "Strong proxy signature and its applications," Proceedings of SCIS, vol. 2001, pp. 603-608, 2001. 
[29] A. Fiat and A. Shamir, "How to prove yourself: Practical solutions to identification and signature problems," in Proc. of Advances in Cryptology CRYPTO86, Springer, pp. 186-194, 1986. Article (CrossRef Link).

[30] U. Feige, A. Fiat, and A. Shamir, "Zero-knowledge proofs of identity," Journal of cryptology, vol. 1, no. 2, pp. 77-94, 1988. Article (CrossRef Link).

[31] F. Zhang and K. Kim, "Id-based blind signature and ring signature from pairings," in Proc. of International Conference on the Theory and Application of Cryptology and Information Security, Springer, pp. 533-547, 2002. Article (CrossRef Link).

[32] K. G. Paterson, "Id-based signatures from pairings on elliptic curves," Electronics Letters, vol. 38, no. 18, pp. 1025-1026, 2002. Article (CrossRef Link).

[33] N. P. Smart, "Identity-based authenticated key agreement protocol based on weil pairing," Electronics letters, vol. 38, no. 13, pp. 630-632, 2002. Article (CrossRef Link).

[34] M. C. Gorantla, R. Gangishetti, and A. Saxena, “A survey on id-based cryptographic primitives.” IACR Cryptology ePrint Archive, vol. 2005, p. 94, 2005.

[35] J. C. Choon and J. H. Cheon, "An identity-based signature from gap diffie-hellman groups," in Proc. of International workshop on public key cryptography, Springer, pp. 18-30, 2003. Article (CrossRef Link).

[36] Q. Wang and Z. Cao, "Efficient id-based proxy signature and proxy signcryption form bilinear pairings," in Proc. of International Conference on Computational and Information Science, Springer, pp. 167-172, 2005. Article (CrossRef Link).

[37] D. Pointcheval and J. Stern, "Security arguments for digital signatures and blind signatures," Journal of Cryptology, vol. 13, no. 3, pp. 361-396, 2000. Article (CrossRef Link).

[38] X. Cao, W. Kou, and X. Du, "A pairing-free identity-based authenticated key agreement protocol with minimal message exchanges," Information Sciences, vol. 180, no. 15, pp. 2895-2903, 2010. Article (CrossRef Link).

[39] S. Namasudra, P. Roy, B. Balusamy, and P. Vijayakumar, "Data accessing based on the popularity value for cloud computing," in Proc. of 2017 International Conference on Innovations in Information, Embedded and Communication Systems (ICIIECS), IEEE, pp. 1-6, 2017. Article (CrossRef Link).

[40] S. Namasudra, P. Roy, P. Vijayakumar, S. Audithan, and B. Balusamy, "Time efficient secure dna based access control model for cloud computing environment," Future Generation Computer Systems, vol. 73, pp. 90-105, 2017. Article (CrossRef Link).

[41] P. Vijayakumar, S. M. Ganesh, L. J. Deborah, S. H. Islam, M. M. Hassan, A. Alelaiwi, and G. Fortino, "Mgpv: A novel and efficient scheme for secure data sharing among mobile users in the public cloud," Future Generation Computer Systems, vol. 95, pp. 560-569, 2019. Article (CrossRef Link).

[42] H.Xiong, Y.Zhao, L.Peng, H.Zhang, and K.H.Yeh, "Partially policy-hidden attribute-based broadcast encryption with secure delegation in edge computing," Future Generation Computer Systems, vol.97, pp. 453-461, 2019. Article (CrossRef Link). 


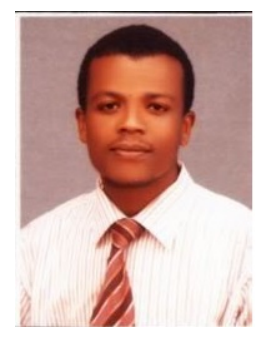

Negalign Wake Hundera is a Ph.D. Candidate at the University of Electronic Science and Technology China (UESTC) since 2017. He obtained his MSc degree in Computer Science and technology from the University of Electronic Science and Technology China (UESTC), Chengdu, China, in 2016 and a Bachelor degree in Information Technology from Jimma University, Jimma, Ethiopia in 2009. His research interest includes network Security, cryptographic protocols, information security, and cloud computing.

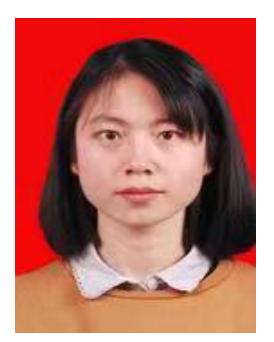

Qian Mei is currently pursuing her Ph.D. degree from the School of Information and Software Engineering, University of Electronic Science and Technology of China. She received her B.S. degree from Jiangxi University of Science and Technology in 2017. Her research interests include certificateless public key cryptography and privacy-preserving.

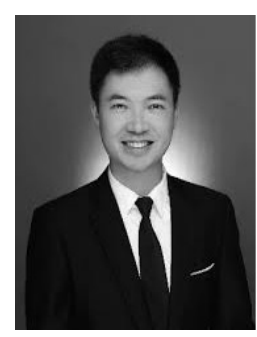

Hu Xiong received the Ph.D. degree from the University of Electronic Science and Technology of China (UESTC) in 2009. He is currently a full Professor at UESTC. His research interests include cryptography and ad hoc network security.

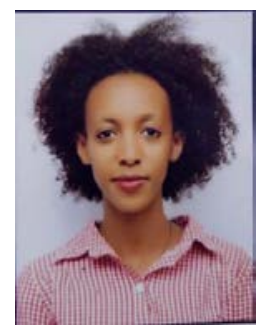

Dagmawit Mesfin Geresu is currently pursuing her bachelor degree in Electronic Information Engineering from the School of Information and Communication Engineering, University of Electronic Science and Technology of China (UESTC). 